University of Nebraska - Lincoln

DigitalCommons@University of Nebraska - Lincoln

$7-2013$

Developing predictive systems models to address complexity and relevance for ecological risk assessment

\author{
Valery E. Forbes \\ University of Nebraska-Lincoln, veforbes@umn.edu \\ Peter Calow \\ University of Nebraska-Lincoln, pcalow2@unl.edu
}

Follow this and additional works at: https://digitalcommons.unl.edu/biosciforbes

Part of the Life Sciences Commons

Forbes, Valery E. and Calow, Peter, "Developing predictive systems models to address complexity and relevance for ecological risk assessment" (2013). Valery Forbes Publications. 55.

https://digitalcommons.unl.edu/biosciforbes/55

This Article is brought to you for free and open access by the Papers in the Biological Sciences at DigitalCommons@University of Nebraska - Lincoln. It has been accepted for inclusion in Valery Forbes Publications by an authorized administrator of DigitalCommons@University of Nebraska - Lincoln. 


\title{
Developing predictive systems models to address complexity and relevance for ecological risk assessment
}

\author{
Valery E Forbes ${ }^{1}$ and Peter Calow ${ }^{2}$ \\ 1. School of Biological Sciences, University of Nebraska-Lincoln, Lincoln, Nebraska 68588-0118 \\ 2. Office of Research and Economic Development, University of Nebraska-Lincoln, Whittier Research Center, Lincoln, Nebraska
}

Corresponding author - V. E. Forbes, vforbes3@unl.edu

\begin{abstract}
Ecological risk assessments (ERAs) are not used as well as they could be in risk management. Part of the problem is that they often lack ecological relevance; that is, they fail to grasp necessary ecological complexities. Adding realism and complexity can be difficult and costly. We argue that predictive systems models (PSMs) can provide a way of capturing complexity and ecological relevance cost-effectively. However, addressing complexity and ecological relevance is only part of the problem. Ecological risk assessments often fail to meet the needs of risk managers by not providing assessments that relate to protection goals and by expressing risk in ratios that cannot be weighed against the costs of interventions. Once more, PSMs can be designed to provide outputs in terms of value-relevant effects that are modulated against exposure and that can provide a better basis for decision making than arbitrary ratios or threshold values. Recent developments in the modeling and its potential for implementation by risk assessors and risk managers are beginning to demonstrate how PSMs can be practically applied in risk assessment and the advantages that doing so could have.
\end{abstract}

Keywords: Ecological relevance, Predictive systems models, Risk management, Risk quotients, Valuation

\section{INTRODUCTION}

Risk assessment of chemicals in the environment is intended for use in risk management, and yet there is evidence that the outputs of risk assessments are not driving risk management decisions (NRC 2009; EC 2013a). One of the reasons for this is that the risk assessments are not providing the kind of information that risk managers need to make their decisions about interventions. Here we explore the basis of this mismatch specifically with regard to the ecological risk assessment (ERA) of chemicals and suggest some remedies that are based on the application of predictive systems models. Our focus is on the European Union (EU) (e.g., Regulation (EC) 1907/2006; Regulation (EC) 1107/2009), where prospective risk assessments dominate, recognizing that the situation in the United States is somewhat different in that site-specific risk assessments of historical contamination have more prominence.

Current methods for ecological risk assessment (ERA) in the EU most often relate simple estimates of predicted or measured exposure concentrations to effects measured in standard toxicity tests conducted under controlled laboratory conditions (Van Leeuwen and Vermeire 2007). Measured effects concentrations are most often divided by some fixed application factor to arrive at an effects threshold (predicted no-effects concentration) that accounts for uncertainties in extrapolating effects from acute to chronic exposure, from the tested species to others, and from laboratory to field. A risk characterization ratio is calculated by comparing the measured or predicted exposure concentration to the effects threshold to give an indication as to whether the threshold of effects is likely to be breached. For pesticides, the toxicity endpoint is divided by the estimated exposure concentration (toxicity exposure ratio [TER]), and this ratio is compared to a predefined threshold value to judge whether risk is acceptable. Occasionally, if there are toxicity data from multiple species, these will be fit to a species sensitivity distribution and a percentile (often the 5th) of the distribution, often with an application factor applied to it, is used as the effects threshold. In rare instances (mostly concerning pesticides) there will be limited results on exposure and effects from mesocosm or semifield studies. However, even in this case, the practice is to define a no-observed effects threshold for comparison with a relevant exposure concentration.

An important feature of the current approach to ERA is that 
it follows a so-called tiered process that starts simply, with worstcase assumptions and seeks to become progressively more realistic at higher tiers (Regulation EC 1907/2006, Regulation EC $1107 / 2009)$. The presumption is that if a chemical passes at a lower tier there is no need for further assessment or intervention. However, chemicals that fail lower tiers are subject to higher tier tests and assessments that presumably are more realistic; failure at the highest tier means that intervention would be required.

Moving up the tiers involves more time, effort, and expense. In the following, we argue that it is possible to improve realism through a modeling approach that does not need to be very expensive or time consuming. Improving realism in this kind of way is necessary, but not sufficient, to enhance the usefulness of ERAs as a basis for risk management. In addition, and to be helpful to managers, ERAs have to be expressed in terms of ecological effects that matter, because they are valued by the public. Rather than being expressed as thresholds (i.e., risk characterization ratios or TERs), ERAs should be expressed in terms of concentration/dose responses (i.e., should quantify how impacts decline with reducing exposure), so that managers can better evaluate the consequences of restrictions on chemicals against the benefits of reduced risk.

\section{CURRENT METHODS FOR ECOLOGICAL RISK ASSESSMENT LACK ECOLOGICAL REALISM}

Although it is widely recognized that ecological variables, such as temperature, food availability, habitat quality, and a host of other factors can potentially influence the risks of chemicals, such influences are routinely ignored in standard ERAs. Although this may be pragmatic for screening-level (i.e., lower tier) assessments, it becomes increasingly problematic for higher tiers that, by definition, are intended to incorporate relevant complexity. A key question is whether adding ecological realism into ERA is likely to increase or decrease risk. The evidence on this question is equivocal, and the literature contains examples of ecological complexities having both positive and negative effects on risk.

An example of ecological complexity increasing risk is seen in the case of indirect effects. In such cases, risk characterization ratios derived from toxic effects of a chemical measured in the species of interest underestimate the likely ecological impacts. A classic example of indirect effects has been documented in an 18-year intensive study of the partridge, Perdix perdix, in Sussex, England (see "Potts, 1986" summarized in Walker et al. [2012]). Significant declines in partridge numbers between approximately 1955 and 1985 were linked with an increase in chick mortality. Different causes for increased chick mortality were proposed, and it was eventually determined that the mortality was caused by a decrease in density of preferred insects that was in turn the result of increased herbicide use reducing the food supply of the insect prey. In this case, a risk characterization ratio based on expected exposure of partridge and direct toxicity of herbicides to them would have indicated a low-risk situation, but actual risks to bird populations would be underestimated if indirect effects were not incorporated into the risk assessment. In practice, ERAs try to address indirect effects by testing toxicity in different taxonomic groups and then basing risk characterization ratios on the most sensitive group. The assumption here is that if risk to the most sensitive group of species is avoided, the chances of indirect effects are minimal. Unfortunately, this is a fairly coarse approach, and there remain substantial uncertainties (e.g., related to untested taxa, sensitivity of tested species to other members of the taxonomic group they are intended to represent, the extent to which the endpoints measured in standard tests are good predictors of relevant impacts, etc.).

An example of ecological complexity decreasing risk is provided by Dalkvist et al. (2009). This study compared risk estimated according to standard EU procedures for pesticide risk assessment for mammals with the output of a sophisticated individual-based model that simulated individual animals moving about in a realistic landscape. An important difference between the 2 risk scenarios is that the standard approach assumes that all individuals in the population are equally exposed to the pesticide, whereas in the model, only that fraction of the population present in the sprayed parts of the landscape is exposed. In this case, predictions from standard procedures overestimate risk because they ignore habitat complexity and assume that all individuals are equally exposed. The analysis also demonstrated that animal ecology can be at least as important in determining risk as the degree of toxicity of the chemical being assessed. An important take-home message from this example is that, given the relative amount of effort that currently goes toward obtaining very precise and repeatable estimates of toxicity versus obtaining ecologically realistic information for the risk scenario (e.g., on exposure, life history of exposed species, etc.) under consideration, it would appear that ERA efforts are being misdirected.

An example of ecological complexity both increasing and decreasing risk involves the influence of density dependence. Most natural populations are believed to be under some kind of density limitation at least for some of the time. There is evidence in the literature of density dependence both increasing (Hayashi et al. 2009) and decreasing (Forbes et al. 2001) risk and even examples of both within the same study depending on the relative strengths of density dependence versus toxicity effects (Linke-Gamenick et al. 1999).

It should not be surprising that adding ecological realism can both increase and decrease risk, depending on the situation and the type of complexity considered. What is more surprising is that we use an approach to ERA that implicitly assumes that using standard application factors can effectively capture the wide range of ecological factors that potentially influence risk. Some have claimed that adding ecological complexities into ERA increases uncertainty (Wang and Luttik 2012). The argument is that including more parameters, each of which is associated with uncertainty, increases the uncertainty of the risk estimates. In a strict technical sense, this would appear to be true as evidenced by widening confidence bounds around model predictions. However, and more importantly, if there are indeed ecological complexities that influence risk, using models that ignore them can result in very precise estimates of risk that are completely wrong. Risk characterization ratios, by being represented as a single number, give the impression that there are no uncertainties, whereas these are hidden in the analyses behind their calculation. Incorporating necessary ecological complexities may make risk estimates more variable, but doing so also makes the sources of uncertainty explicit and ideally can be used to distinguish uncertainty from variability. Ideal risk assessments would be both precise and accurate. Our point is that risk quotients, although appearing very precise, hide uncertainties - and they may also be inaccurate. We believe that making the uncertainties explicit is helpful and that, whereas we want to reduce uncertainties (e.g., from lack of understanding), we do need 
to capture sources of variability (e.g., among species) that matter for the risk assessment.

This is not to imply that we have to add all possible ecological complexities to estimate risk effectively. The challenges are to decide which and how much complexity to include and to clearly distinguish variability from uncertainty. The bottom line is that ERA needs to incorporate complexities in a way that is efficient, robust, transparent, understandable, and that makes biological sense.

\section{CURRENT ECOLOGICAL RISK ASSESSMENT OUTPUTS ARE FAR REMOVED FROM PROTECTION GOALS AND MANAGEMENT NEEDS}

We make 3 points in this section: first that the thresholds used in ERAs are not obviously related to ecologically relevant endpoints; second, even if they were, there still needs to be an explicit connection between the endpoints and the ecological protection goals; and third, even if that is fulfilled, they still need to be expressed on a continuous scale of concentration-dose-response.

\section{Endpoints used to estimate thresholds are not ecologically relevant}

A key question is, "what is the relationship between risk characterization ratios and impacts on ecological protection goals?" Unfortunately, there is a substantial body of literature that would indicate that we either do not know, the relationship is very uncertain, and/or the relationship varies widely across categories of test species and environmental compartments. Thresholds derived from standard ecotoxicological tests that go into ERAs are in terms of the responses of individual organisms to toxicants (i.e., their survival, reproduction, and growth). What matters from an ecological point of view is the likely impact on populations (to ensure persistence in space and time) and on ecosystem structure and processes. The literature shows that the level of protection at a population level varies widely among toxicants, which means that risk characterization ratios (or TERs) are not consistently protective.

Luttik et al. (2011) showed that the level of protection offered by first-tier pesticide risk assessments varies widely among pesticides. For crustaceans, an average of $3.4 \%$ of species would be exposed above their median lethal concentration in $10 \%$ of receiving surface waters that receive the maximum allowable exposure to an individual pesticide. Furthermore, this percentage varied widely among different pesticides and reached a maximum of $41.4 \%$ of species. A similar analysis for birds resulted in somewhat different values. This analysis clearly demonstrates that the degree of protection offered by standard first-tier assessments is highly variable-among chemicals and among taxonomic groups. Hanson and Stark (2012) demonstrated a huge amount of uncertainty in the ability of standard acute and chronic endpoints to predict population-level risks. Part of this kind of variability can be explained by common effects at the individual level in different species translating into different effects at the population level due to life-history differences (Calow et al. 1997). A comprehensive analysis comparing thresholds derived from species sensitivity distributions to effects of pesticides observed in mesocosm studies (Maltby et al. 2009) has shown that, whereas SSD thresholds may be protective if compared to mesocosm effects, they are not at all predictive, thus demonstrating that the degree of protection offered by current ERA approaches is not at all consistent.
Endpoints need to connect to protection goals

Risk managers have the responsibility of intervening to ensure that ecological properties that matter for the public are protected to an extent that is reasonable economically. One way of capturing protection goals is in terms of impacts on ecosystem services that, by definition, matter and are valued (Forbes and Calow 2012aa). That being so, it becomes important to make explicit quantitative connections between the ecological endpoints that are studied in ERA and these ecosystem services. Although some steps are being made in this direction in both Europe (EFSA PPR 2010) and the United States (Munns et al. 2009), there is much more that can and should be done in this area.

\section{Thresholds are a blunt tool for risk managers}

Ideally, risk management needs not only to know under what circumstances to intervene but to what extent. Otherwise potential benefits associated with the use of chemicals may be lost, and resources used for intervention may be unnecessarily spent. Thresholds are a flimsy basis for this kind of risk management for 2 reasons. First, they can encourage no intervention (below threshold) or complete banning (above threshold). Second, to move beyond this all-or-nothing situation, judgments are often made on the seriousness of effects above thresholds and the extent to which these should be managed. Because this is usually a nontransparent process, it is hard to know how extensive it is, but it is clear that professional judgments about the seriousness of risks are widely used in ERA and can involve both risk assessors and risk managers.

The consequence of all of the above is that results of ERAs are not driving management decisions in the way that they should (EC 2013a), yet the amount of resources spent on risk assessments in various legislative contexts is high. Why put so much effort into ERA if the output is not going to explicitly inform decision making? The implication is that either such resources could be better spent or that substantial improvements are needed in ERA for it to provide information on which risk managers can confidently rely.

\section{PREDICTIVE SYSTEMS MODELS OFFER A PROMISING TOOL FOR ERA}

Trying to develop approaches to ERA that overcome the weaknesses of the current approach is going to be very challenging. Although there is no silver bullet, we believe that predictive systems models (PSMs) can provide a tractable basis by incorporating necessary ecological realism, by developing ecological production functions (to link responses of organisms to chemicals to impacts on ecosystem service delivery), and by providing concentration/ dose-response relationships (Forbes and Calow 2012bb). Predictive systems models are models based on mechanistic understanding of key processes and in which properties at higher levels of biological organization emerge from processes operating at lower levels of organization.

Predictive systems models can provide a sounder scientific basis for addressing the extrapolation problems currently approached with the use of uncertainty factors. Predictive systems models provide abstractions of real systems yet represent processes and their consequences across levels of biological organization in a mechanistic way. For example, toxicokinetic-toxicodynamic (TKTD) models link exposure with effects through detailed information on toxicokinetics (i.e., uptake, distribution, biotransformation, and elimination), and toxicodynamics (i.e., interaction of toxicant with 
its target site). They are used to extrapolate toxicity among species and across different exposure scenarios (Kretschmann et al. 2012). Energy budget models represent the uptake of energy into organisms and use in the various aspects of metabolism from maintenance to growth and reproduction. In this way they can represent the different physiologies of organisms and can provide a mechanistic basis for extrapolating effects that involve energy metabolism and its linkage to life-history outcomes across species (Sibly et al. 2013). Demographic models, such as matrix and metapopulation models, integrate information on individual age- or stagedependent survival and reproduction to effects on population dynamics. They can be used to extrapolate toxicant effects from the individual to population level and to extrapolate across different life cycles (Forbes et al. 2008). Agent- or individual-based models (ABMs) are the newest and most flexible type of PSM with potential for use in ERA. Agent-based models describe individual organisms (agents) as discrete and unique entities that interact with each other and their environment. The advantages of ABMs are that they are not limited by mathematical tractability, they can incorporate spatial and temporal variability, they can capture interactions among species (e.g., through changes in food supply or predation that may result in indirect effects), they can capture interactions among multiple stressors, and they are ideal for studying phenomena that cross levels of biological organization and properties that emerge from interactions of the parts within the system. In this way, ABMs can facilitate mechanistic extrapolation from simplified laboratory tests to more realistic field situations, extrapolate from acute to chronic effects, and, with adjustment of certain model parameters, can be used to extrapolate across species.

For example, an important concern in risk assessment is how spatial variability in key habitat features can influence exposure to, and hence effects of, chemicals in populations. Meli et al. (2013) developed an ABM to explore how various patterns of spatial heterogeneity in soil contaminant levels, in combination with avoidance behavior, could influence risk to collembolan populations from contaminated soil. Liu et al. (2013) developed an ABM to examine how typical landscape dynamics and the schedule of pesticide application can influence pesticide risk to wood mouse, Apodemus sylvaticus, populations in realistic agricultural landscapes.

Another important question for ERA is how chemical impacts (alone and in combination with other stressors) on organism physiology result in effects on individual life-history traits and population dynamics. Martin et al. (2012) developed a generic ABM that is based on Dynamic Energy Budget (DEB) theory. The model can be used to link impacts on energy allocation processes to life history and population dynamics, to explore influences of environmental variables such as food density, and can incorporate spatial effects (e.g., variability in food supply or exposure to toxicants) on individuals and populations.

Yet another issue that is often of concern in ERA is how differences in exposure scenarios, such as timing and time course of exposure, are likely to influence risk. This is extremely difficult to address empirically because of time and cost constraints and is an obvious question to address with PSMs. For example, Wang and Grimm (2010) developed a structurally realistic but relatively simple ABM of the common shrew (Sorex araneus) to test how landscape structure and the timing of pesticide application can influence risk to shrew populations. They also used the model to assess the relative sensitivity of different population-level endpoints for detecting risk. TKTD models are ideal for predicting effects from time varying exposures (Nyman et al. 2012), and could be linked with ABMs to explore population-level impacts of this kind of realistic exposure scenario.

The above are just a few examples to demonstrate how PSMs can improve the ecological realism of ERA and address concerns of risk assessors and risk managers that would be extremely difficult, if not impossible, to study using empirical approaches. The usefulness of these PSMs is not only in generating concrete estimates of risk, but in providing insights into which ecological factors are most likely to ameliorate or aggravate risk. As such, they can provide an important source of hypotheses for designing focused studies and a useful background for risk management.

Most of the advances in PSMs for ERA have been developed in the context of pesticide risk assessment. This is likely due to a combination of more toxicity test data being available, a more restricted set of exposure scenarios and landscape types to consider, and a greater consensus on focal species of concern than there is for other chemical classes. However, the benefits that PSMs could bring to ERA of other chemical classes is no less, and indeed these models can offer powerful tools for assessing risks of other human activities and impacts (e.g., habitat destruction, climate change, etc.) on ecological systems.

\section{CHALLENGES IN MODEL DEVELOPMENT AND IMPLEMENTATION}

Of course, there are challenges for model development and implementation. Many in the risk assessment/management community are not familiar with PSMs. Particularly ABMs can incorporate a lot of complexity and can therefore suffer from significant "black box" issues. Even if the ABMs are described transparently (Grimm et al. 2010), their great flexibility means that it takes more effort (compared to, for example, matrix models) to understand what the model is doing and why. Often, there is a lack of basic ecological information (life-history features and behavior) for parameterizing PSMs for particular species. This underlines the need for more basic ecological research for focal species to use in PSMs.

Over and above improving the science to enhance model development, there is a need for substantial stakeholder involvement to facilitate effective model implementation. For example, it would be beneficial for ERA purposes to have consensus on modeling platform, communication of model features, extrapolation issues, and questions of scale. Last but not least, we need to have agreement on quantifiable protection goals before we can develop models to predict impacts on them. Although such discussions are contentious and difficult, they are a necessary prerequisite to developing a sound approach to ERA.

Significant progress is being made on the incorporation of PSMs (sometimes called mechanistic effect models) into ERA. Several workshops have been organized with the intent to bring together diverse groups of stakeholders to explore the barriers and solutions to the use of such models in ERA (i.e., LEMTOX [Thorbek et al. 2010], RUC09 [Forbes et al. 2011], and Modelink [Hommen 2013]). An advisory group (MEMoRisk) has been established under SETAC Europe to explore and evaluate the benefit of mechanistic effect modeling for the risk assessment of chemicals in Europe and elsewhere (Preuss et al. 2009). The European Union has funded a major Marie Curie Initial Training Network (CREAM, http://cream-itn.eu/) (Grimm et al. 2009) to train the next generation of ecological modelers. CREAM is developing 
and experimentally validating a suite of mechanistic effect models for organisms relevant for chemical risk assessments. An important goal of the network is to formulate guidance for good modeling practice (Schmolke et al. 2010) that emphasizes consistency, transparency and rigorous model evaluation as core elements of the modeling process. And finally, 2 important opinions produced by expert groups of European Union bodies highlight a significant role for modeling in the future of ERA (EFSA PPR 2010, EC 2013a).

\section{CONCLUSIONS}

The tiered approach to ERA implies that the estimated risk should reduce from lower to higher tiers on the presumption that the lower tiers are sufficiently conservative. This means that adding more ecological realism is likely to reduce the estimates of risk, but as indicated above this may not always be the case. Engaging in higher tiered assessments is expensive and takes time, and it is hard to judge if the effort will be worthwhile because the reduction is unpredictable; sometimes it is small, and sometimes it is large (e.g., SSD vs mesocosm comparisons) (Maltby et al. 2009). We have argued that the PSM approach is a way of exploring the effects of ecological complexity on risk for less expense and in less time in particular circumstances. In principle, PSMs could in some cases replace existing higher-tier studies and in other cases focus testing efforts on those factors that are most likely to influence risk.

Vighi (2012) called for approaches "capable of answering more complex questions than dose/concentration- response relationships can." We agree that we need to move beyond standard laboratory toxicity tests as the basis of ecological effects assessments. However, we believe that coming to terms with ecological complexity is only part of the challenge and that effective risk management requires being able to calibrate changes in adverse effects with changes in chemical exposure. This underlines the fact that assessments based on thresholds of effects are not helpful for risk management. Also making endpoints more value relevant is key if ERAs are to provide a basis for interventions that relate to effects that matter to the public and that can be weighed against the costs of interventions.

Much has been written over the years about the failure of ERA to take account of ecological complexity, and there has been recognition that improvements in risk assessment for the sake of better management are required (NRC 2009; EC 2013b). We believe that progress is now possible because of developments in the modeling and ongoing efforts to promote dialogue among risk assessors, risk managers and modelers on the key issues. The challenge here will be to establish forums for dialogue that allow information exchange about what is valued for protection without allowing values to bias the analyses and conclusions (Calow and Forbes 2010).

\section{Acknowledgments}

This article is based on an invited presentation given by V Forbes entitled, "Addressing complexity in ecological systems - ignore it or embrace it?" as part of the Special Symposium on New Challenges for Ecological Risk Assessment, at the 6th SETAC World Congress, 20-24 May 2012, Berlin, Germany. The authors thank M Vighi for inviting this work to be presented. This work and the views expressed here have been inspired by interactions with our many collaborators and students. Most notably we acknowledge participants in the European Union 7th Framework Program Project, CREAM (PITN-GA-2009-238148).

\section{REFERENCES}

Calow P, Forbes VE. 2010. Ecological risk assessment should be value-relevant but not value-biased. Integr Environ Assess Manag 6:784-785.

Calow P, Sibly R, Forbes VE. 1997. Risk assessment on the basis of simplified population dynamics' scenarios. Environ Toxicol Chem 16:1983-1989.

Dalkvist T, Topping CJ, Forbes VE. 2009. Population-level effects of pesticide-induced chronic effects on individuals depend more on ecology than toxicology. Ecotoxicol Environ Saf 72:1663-1672.

[EC] European Commission. 2013a. Making risk assessment more relevant for risk management. Opinion of the Scientific Committee on Health and Environmental Risks (SCHER) and the Scientific Committee on Emerging and Newly Identified Health Risks (SCENIHR). [cited 2013 January]. Online: http://ec.europa.eu/health/scientific committees/consumer safety/docs/sccs o 130.pdf

[EC] European Commission. 2013b. Addressing the new challenges for risk assessment. Joint Opinion of SCHER (Scientific Committee on Health and Environmental Risks), SCENIHR (Scientific Committee on Emerging and Newly Identified Health Risks), SCCS (Scientific Committee on Consumer Safety) of the European Commission, Brussels. [cited 2013 January]. Online: http://ec.europa.eu/health/ scientific committees/consumer safety/docs/sccs o 131. pdf

[EFSA PPR] European Food Safety Authority Panel on Plant Protection Products and Their Residues. 2010. Scientific opinion on the development of specific protection goal options for environmental risk assessment of pesticides, in particular in relation to the revision of the Guidance Documents on Aquatic and Terrestrial Ecotoxicology (SANCO/3268/2001 and SANCO/10329/2002). EFSA J 8: 1821.

Forbes VE, Calow P, Sibly RM. 2008. The extrapolation problem and how population modeling can help. Environ Toxicol Chem 27:1987-1994.

Forbes VE, Calow P. 2012a. The use of the ecosystem services concept in ecological risk assessment. Integr Environ Assess Manag 9:269-275.

Forbes VE, Calow P. 2012b. Promises and problems for the new paradigm for risk assessment and an alternative approach involving predictive systems models. Environ Toxicol Chem 31:2663-2671.

Forbes VE, Calow P, Grimm V, Hayashi TI, Jager T, Katholm A, Palmqvist A, Pastorok R, Salvito D, Sibly RM., et al. 2011. Adding value to ecological risk assessment with population modeling. Hum Ecol Risk Assess 17:287-299.

Forbes VE, Sibly RM, Calow P. 2001. Toxicant impacts on density-limited populations: a critical review of theory, practice and results. Ecol Appl 11:1249-1257.

Grimm V, Ashauer R, Forbes V, Hommen U, Preuss TG, Schmidt A, van den Brink PJ, Wogram J, Thorbek P. 2009. CREAM: A European project onmechanistic effect models for ecological risk assessment of chemicals. Environ Sci Pollut Res 16:614-617.

Grimm V, Berger U, DeAngelis DL, Polhill G, Giske J, Railsback SF. 2010. The ODD protocol: A review and first update. Ecol Model 221:2760-2768. 
Hanson N, Stark JD. 2012. Comparison of population level and individual level endpoints to evaluate ecological risk of chemicals. Environ Sci Technol 46:5590-5598.

Hayashi TI, Kamo M, Tanaka Y. 2009. Population-level ecological effect assessment: Estimating the effect of toxic chemicals on density-dependent populations. Ecol Res 24:945-954.

Hommen U. 2013. First MODELINK workshop successfully held in Le Croisic, France. SETAC. Globe 14:1.

Kretschmann A, Ashauer R, Hollender J, Escher BI. 2012. Toxicokinetic and toxicodynamic model for diazinon toxicityMechanistic explanation of differences in the sensitivity of Daphnia magna and Gammarus pulex. Environ Toxicol Chem 31:2014-2022.

Linke-Gamenick I, Forbes VE, Sibly RM. 1999. Density-dependent effects of a toxicant on life-history traits and population dynamics of a capitellid polychaete. Mar Ecol Prog Ser 184:139-148.

Liu C, Sibly RM, Grimm V, Thorbek P. 2013. Linking pesticide exposure and spatial dynamics: An individual-based model of wood mouse (Apodemus sylvaticus) populations in agricultural landscapes. Ecol Model 248:92-102.

Luttik R, Hart A, Roelofs W, Craig P, Mineau P. 2011. Variation in the level of protection afforded to birds and crustaceans exposed to different pesticides under standard risk assessment procedures. Integr Environ Assess Manag 7:459-465.

Maltby L, Brock TCM, Van den Brink PJ. 2009. Fungicide risk assessment for aquatic ecosystems: Importance of interspecies variation, toxic mode of action, and exposure regime. Environ Sci Technol 43:7556-7563.

Martin BT, Zimmer EI, Grimm V, Jager T. 2012. Dynamic Energy Budget theory meets individual-based modeling: A generic and accessible implementation. Methods Ecol Evol 3:445-449.

Meli M, Auclerc A, Palmqvist A, Forbes VE, Grimm V. 2013. Population-level consequences of spatially heterogeneous exposure to heavy metals in soil: An individual-based model of Springtails. Ecol Model 250:338-351.

Munns WR Jr, Helm RC, Adams WJ, Clements WH, Cramer MA, Curry M, DiPinto LM, Johns DM, Seiler R, Williams LL., et al. 2009. Translating ecological risk to ecosystem service loss. Integr Environ Assess Manag 5:500-514.

[NRC] National Research Council. 2009. Science and decisions. Washington, DC: National Academy of Science Publications.

Nyman A-M, Schirmer K, Ashauer R. 2012. Toxicokinetic-toxicodynamic modeling of survival of Gammarus pulex in multiple pulse exposures to propiconazole: Model assumptions, calibration data requirements and predictive power. Ecotoxicology 21:1828-1840.
Preuss TG, Hommen $U$, Alix A, Ashauer R, van den Brink $P_{\text {, }}$ Chapman P, Ducrot V, Forbes VE, Grimm V, Schäfer D., et al. 2009. Mechanistic effect models for ecological risk assessment of chemicals (MEMoRisk)-A new SETAC-Europe Advisory Group. Environ Sci Pollut Res 16:250-252.

Regulation (EC) No 1907/2006 of the European Parliament and of the Council of 18 December 2006 concerning the Registration, Evaluation, Authorisation and Restriction of Chemicals (REACH), establishing a European Chemicals Agency, amending Directive 1999/45/EC and repealing Council Regulation (EEC) No 793/93 and Commission Regulation (EC) No $1488 / 94$ as well as Council Directive 76/769/EEC and Commission Directives 91/155/EEC, 93/67/EEC, 93/ 105/ EC and 2000/21/EC.

Regulation (EC) No 1107/2009 of the European Parliament and of the Council of 21 October 2009 concerning the placing of plant protection products on the market and repealing Council Directives 79/117/EEC and 91/414/EEC.

Schmolke A, Thorbek P, DeAngelis DL, Grimm V. 2010. Ecological models supporting environmental decision making: A strategy for the future. Trends Ecol Evol 25:479-486.

Sibly RM, Grimm V, Martin BT, Johnston ASA, Kułakowska K, Topping CJ, Calow P, Nabe-Nielsen J, Thorbek P, DeAngelis DL. 2013. Representing the acquisition and use of energy by individuals in agent-based models of animal populations. Methods Ecol Evol 4:151-161.

Thorbek P, Forbes V, Heimbach F, Hommen U, Thulke H-H, Van den Brink PJ, Wogram J, Grimm V, editors. 2010. Ecological models for regulatory risk assessments of pesticides: Developing a strategy for the future. Society of Environmental Toxicology and Chemistry (SETAC). Boca Raton (FL): CRC. 127 p. Van Leeuwen CJ, Vermeire TG. 2007. Risk assessment of chemicals: An introduction, 2nd ed. Dordrecht, the Netherlands: Springer. 686 p.

Vighi M. 2012. Berlin Special Session-New challenges for ecological risk assessment. SETAC. Globe 13:1-4.

Walker CH, Sibly RM, Hopkin SP, Peakall DB. 2012. Principles of ecotoxicology, 4th ed. Boca Raton (FL): CRC. 360 p.

Wang M, Grimm V. 2010. Population models in pesticide risk assessment: Lessons for assessing population-level effects, recovery, and alternative exposure scenarios from modeling a small mammal. Environ Toxicol Chem 29:1292-1300.

Wang M, Luttik R. 2012. Population level risk assessment: Practical considerations for evaluation of population models from a risk assessor's perspective. Environ Sci Eur 24:3. 